\title{
COVID-19 severity and in-hospital mortality in an area with high HIV prevalence
}

Michael T Boswell ( $\nabla$ boswell.michaelt@gmail.com )

University of Pretoria https://orcid.org/0000-0003-2152-1617

Tshegofatso Maimela

University of Pretoria

Dan Hameiri-Bowen

Oxford University

George Riley

University of Pretoria

Albertus Malan

Tshwane District Hospital

Nickietta Steyn

University of Pretoria

Nomonde Nolutshungu

University of Pretoria

Talita De Villiers

Tshwane District Hospital

Zelda de Beer

University of Pretoria

Tshilidzi Maselesele

Steve Biko Academic Hospital

Universe Masoma

Steve Biko Academic Hospital

Mantwa Tolo

Steve Biko Academic Hospital

Katlego Boshielo

Steve Biko Academic Hospital

Bongani Mashaba

Steve Biko Academic Hospital

\section{Simon Spoor}

Tshwane District Hospital

\section{Fareed Abdullah}

South African Medical Research Council

Rajiev Ramlall 
Tshwane District Hospital

\section{Marthinus Heystek}

Tshwane District Hospital

\section{Debashis Basu}

Steve Biko Academic Hospital

\section{Paul Rheeder}

University of Pretoria

\section{Theresa M Rossouw}

University of Pretoria https://orcid.org/0000-0003-4066-922X

Veronica Ueckermann

University of Pretoria https://orcid.org/0000-0002-4419-3583

Wesley van Hougenhouck-Tulleken ( $\square$ westulleken@gmail.com )

University of Pretoria https://orcid.org/0000-0003-4625-0139

\section{Research Article}

Keywords: HIV, COVID-19, South Africa, SARS-CoV-2, mortality

Posted Date: December 9th, 2021

DOI: https://doi.org/10.21203/rs.3.rs-1156502/v1

License: (c) (1) This work is licensed under a Creative Commons Attribution 4.0 International License.

Read Full License 


\section{Abstract}

Background: HIV is moderate risk factor for developing severe COVID-19 and is associated with increased risk of COVID-19 mortality. HIV infection causes immune dysregulation characterised by progressive lymphopenia, chronic immune activation, immunological senescence, and T cell exhaustion. These changes are partly reversed by effective antiretroviral therapy (ART), which reduces morbidity and mortality in people living with HIV (PWH). We investigated the associations among clinical phenotypes, laboratory biomarkers, and hospitalisation outcomes in a cohort of people hospitalised with COVID-19 in a high HIV prevalence area.

Methods: We conducted a prospective observational cohort study in the Tshwane District Hospital complex in Pretoria, South Africa. We analysed data for patients admitted from April to November 2020, before the SARS-CoV-2 Beta variant-driven second wave. Respiratory disease severity was quantified using the respiratory oxygenation (ROX) score. Analysed biomarkers included full blood counts, differential white cell counts, C-reactive protein (CRP), ferritin, procalcitonin (PCT), D-dimer (DDIM), creatinine, alanine aminotransferase (ALT), CD4 T cell counts, and HIV-1 viral loads (HIVVL).

Results: The analysis included 558 patients, of whom 112 (21.7\%) died during admission. The mean age of the cohort was 54 (SD \pm 16 ) years, and numbers of males (50.5\%) and females (49.5\%) were equivalent. A total of $82(15 \%)$ were HIV-positive. PWH were younger (mean age 46 years) than HIVnegative people; most were on ART with a suppressed HIVVL (72\%) and the median CD4 count was 159 (IQR 66-397) cells/ $\mu \mathrm{L}$ at the time of admission. After adjusting for age, HIV was not associated with significantly increased risk of mortality during hospitalisation (aHR=1.1, 95\% Cl: 0.6-2.0). Levels of supportive care were similar in HIV-negative patients and PWH. Inflammatory biomarker levels were equivalent in PWH and HIV-negative patients. A total of 15 PWH had detectable HIVVLs (>1000 copies/mL). Detectable HIVVL was associated with higher ROX scores - indicating less severe respiratory disease. In PWH, mortality was associated with higher levels of CRP, ferritin, PCT and DDIM. When compared to HIV-negative patients who died, PWH who died were younger, had higher DDIM levels, and were more likely to have tuberculosis.

Conclusions: HIV per se was not associated with substantively increased risk of severe disease, or inhospital mortality from COVID-19. Respiratory disease was less severe in PWH with detectable HIVVL. Inflammatory biomarker levels were equivalent in PWH and HIV-negative people, regardless of HIVVL. Increased levels of inflammatory biomarkers and DDIM were associated with in-hospital mortality irrespective of HIV status.

\section{Introduction}

People living with HIV (PWH) have an increased risk of mortality from infection with respiratory viruses including influenza and human metapneumovirus $(1,2)$. Many studies have reported that $\mathrm{PWH}$, especially those not on antiretroviral therapy (ART) and with a detectable HIV-1 viral load (HIVVL), have a higher risk of COVID-19 related in-hospital mortality (3-5). However, observational cohort studies of hospitalised 
patients with COVID-19 have reported that PWH had lower oxygen requirements during their admission. In these studies, patients with detectable HIVVL had lower relative risk of intubation than PWH with suppressed HIVVL $(6,7)$. In a randomised controlled trial, PWH had similar outcomes after initiation of high-flow nasal oxygen or ventilatory support (8). There is still some uncertainty as to whether COVID-19 immunopathology and clinical phenotypes are altered by HIV coinfection.

Current evidence suggests that severe COVID-19 is associated with dysregulation of the monocytemacrophage response, defective $T$ cells responses, elevated inflammatory cytokines, and hyperactivated neutrophils which culminate in ongoing, inappropriate systemic inflammation which damages pulmonary and other tissues (9-12). People hospitalised with COVID-19, particularly with the more severe spectrum of disease, can develop acute respiratory distress syndrome (ARDS) which is associated with systemic inflammation (13). Established markers of respiratory disease severity in COVID-19 include respiratory oxygenation (ROX) scores and $\mathrm{PaO}_{2} / \mathrm{FiO}_{2}$ ratios $(8,13,14)$. Treatments which reduce mortality in COVID19 are anti-inflammatory in nature, with either broad and non-specific targets, like high dose corticosteroids, or targeted, like the interleukin 6 inhibitor, tocilizumab (15).

HIV infection has strong effects on cellular immune phenotypes and function, affecting $T$ and $B$ lymphocytes, and monocytes - all of which are implicated in COVID-19 pathophysiology (16-18). Recently, more evidence has emerged to assess the effect of HIV on cellular immune responses on COVID-19. HIV coinfection does not appear to alter SARS-CoV-2 CD4+ function or phenotypes, but is associated with reduced CXCR3 expression on CD8+ T cells $(19,20)$. Higher HIVVLs are also associated with increased expression of activation markers on CD8+ T cells in COVID-19, which may alter disease manifestations. PWH mount similar SARS-CoV-2 specific antibody responses in acute COVID-19 as HIVnegative people (21). HIV viraemia alters monocyte subpopulation phenotypes, reducing CCR2 and CX3CR1 expression, which may affect their ability to move from blood into tissue. In COVID-19 this may reduce pulmonary inflammation (18).

The majority of PWH analysed in larger cohorts or systematic reviews were on ART, with suppressed HIVVL and high CD4 T cell counts $(3,4,22)$. Consequently, the effect of HIV viraemia and associated immunological changes on the clinical manifestations of COVID-19 remain poorly described. Uncertainty also exists as to the extent to which comorbidities in PWH, such as hypertension, diabetes, or opportunistic infections, contribute to the increased risk of morbidity and mortality.

We investigated whether HIV infection is associated with COVID-19 severity, differences in routinely collected laboratory biomarkers, and mortality after hospitalisation in a well-characterised clinical cohort of patients admitted with COVID-19, in a setting with a high HIV prevalence. Doing so contributes to a better understanding of COVID-19 clinical phenotypes in PWH.

\section{Methods}

\section{Cohort description}


We conducted a prospective, single centre, observational cohort study of patients admitted to the Tshwane District Hospital Complex (TDH) from April to November 2020. This period encompasses the first COVID-19 wave in Pretoria and predates the widespread prevalence of the SARS-CoV-2 Beta/B.1.351 variant which occurred during the second wave from December 2020 to February 2021. This hospital complex was the primary referral centre for COVID-19 cases in the greater Tshwane area, encompassing a population of approximately three million people, with a HIV prevalence of $10.5 \%$ (95\% confidence interval [Cl] $7.7-14.1 \%)$ in $2017(23,24)$. The hospital could admit 66 adults to general ward care levels (WHO COVID-19 severity score 3 - 4), a dedicated COVID-19 High Care unit (22 adult beds) and an ICU (10 adult beds). The High Care unit admitted patients in need of dialysis, close monitoring on oxygen, high flow nasal oxygen and non-invasive ventilation (WHO score $4-5$ ). The ICU was used for patients who were intubated and ventilated (WHO score 6 - 7).

Data including demographic information, comorbidities, date of admission, symptom onset, date of SARS-CoV-2 PCR test, vital signs, level of care, and admission outcome were captured on standardised case report forms (CRF). The CRFs were completed by treating clinicians during patients' admission. Data from the CRFs were entered by research assistants into a REDCap database hosted by the University of Pretoria and reviewed by clinicians involved in the study for accuracy. Hospital admission outcome was coded as survived or died. Survived included patients transferred to other hospitals for further medical care after discharge from the COVID-19 units. Died would include patients with confirmed deaths during hospitalisation for COVID-19. We analysed age as a continuous variable and additionally stratified the cohort into age groups with 20 year-increments.

Admission vital sign data were taken as the worst score within a 48-hour window around the date of admission (admission \pm 24 hours). The respiratory oxygenation (ROX) score was calculated for participants with admission vital data (Figure S1) (14). The ROX score is a continuous variable which estimates respiratory disease severity by creating a composite score considering the supplemental oxygen concentration, peripheral oxygen saturation, and respiratory rate.

Laboratory biomarker data were extracted from the South African National Health Laboratory Services (NHLS) online data warehouse. We analysed haematology panels (full blood count; differential white cell counts including absolute neutrophil count [ANC], lymphocyte count [ALC], neutrophil-to-lymphocyte ratio [NLR]); organ function biomarkers (creatinine, alanine aminotransferase [ALT]); inflammatory biomarkers (C-reactive protein [CRP], ferritin, procalcitonin [PCT]); and D-dimer (DDIM). Laboratory biomarkers were aggregated as median values for admission \pm 24 hours. We analysed CD4+ T cell counts taken during admission. CD4+ T cell counts were stratified at 200 cells/ $\mu \mathrm{L}$ into higher (equal to, or above, $200 \mathrm{cells} / \mu \mathrm{L}$ ) and lower (below 200 cells/ $\mu \mathrm{L}$ ) CD 4 counts. Plasma HIVVL was assessed from the previous 12 months and during admission, with the most recent value being used for this analysis. HIVVLs were stratified at 1000 copies $/ \mathrm{mL}$ into detectable (equal to, or above, 1000 copies $/ \mathrm{mL}$ ) and suppressed (below 1000 copies $/ \mathrm{mL})$.

\section{Statistical analysis}


Data were analysed in R studio (25-29). Baseline characteristics of participants were summarised as means and standard deviations (SD), medians and interquartile ranges (IQR), and counts with percentages as appropriate. Pairwise comparisons of continuous variables were done using MannWhitney (MW) U tests or T tests, dependent on the variables' distribution. Comparison of proportions was performed using chi-squared tests, or fishers exact test. Correlations were analysed by Spearman Rank or Pearsons correlation coefficients - dependent on the variable's distribution. The sensitivity and specificity of variables' ability to predict higher levels of supportive care were calculated using Area Under the Receiver Operating Characteristic curve (AUROC). An AUROC cut-off of 0.7 was used to decide if a variable had good predictive ability for a specified outcome.

The association between age and comorbidities was analysed by logistic regression models, with age treated as a continuous variable. Univariate survival analysis was analysed by Kaplan-Meier survival curves with hypothesis testing via log rank tests. Multivariable survival analysis was done using Cox regression models. Schoenfield residuals were used to test for violation of the proportional hazard's assumption. Markers of disease severity, including ROX scores, and laboratory biomarkers were used to stratify the cohort into mutually exclusive tertiles of comparable size.

\section{Ethical approval}

Ethics approval was granted by the University of Pretoria's Faculty of Health Sciences Research Ethics Committee, and permission obtained from the institutional authorities to collect clinical data from patients admitted to the Tshwane District Hospital complex. Protocol ethics reference number: 637/2020.

\section{Results}

\section{Cohort description}

A total of 558 patient records were analysed (Table 1). The mean age of this cohort was 54 (standard deviation $[S D] \pm 16$ ) years with equivalent numbers of male $(49.5 \%)$ and female $(50.5 \%)$ patients. Hypertension and diabetes were the most common comorbidities, at $55 \%$ and $41 \%$ respectively. Younger patients were more likely to be HIV-positive at admission (Age and HIV: OR = 0.96, 95\% Cl: 0.94-0.97, $P<0.001$ ), and older patients were more likely to have non-communicable comorbidities including hypertension (OR $=1.07,95 \% \mathrm{Cl}: 1.06-1.09, P<0.001)$, diabetes $(\mathrm{OR}=1.03,95 \% \mathrm{Cl}: 1.02-1.04, P<0.001)$, cardiovascular disease $(\mathrm{OR}=1.04,95 \% \mathrm{Cl}: 1.03-1.06, P<0.001)$ and chronic kidney disease $(\mathrm{OR}=1.02$, $95 \% \mathrm{Cl}: 1.0-1.04, P=0.03$ ) (Figure 1).

A total of $82 \mathrm{PWH}$ were admitted during this period (15\% of cohort). PWH were younger than HIV-negative patients and were less likely to have hypertension or CVD (Table 2). PWH were more likely to have a previous, or current, diagnosis of tuberculosis (TB), but the number of patients with active TB was small $(n=14)$. CD 4 counts were available for $56(68.3 \%)$ patients, and the median CD4 count was 159 (IQR: 66397) cells/ $\mu \mathrm{L}$. A total of 32/56 (61.5\%) PWH had CD 4 counts below 200 cells/ $\mu \mathrm{L}$. HIVVLs were available for 52 (63.4\%), and the median HIVVL was 59789 (IQR: 9 417-194 534) copies/mL. HIVVLs were 
suppressed in $37 / 52(71.2 \%)$. The median duration of symptoms before admission was slightly longer in PWH (8.4 days vs 7.1 days, $P=0.04$ ). There was no difference in the proportion of symptoms reported at admission, with cough and dyspnoea the most common (Figure 2) (Chi-squared $P>0.05$ for all comparisons).

Table 1: Cohort demographics, admission vital signs and biomarker levels 
COVID-19 hospital cohort $(n=558)$

Missing n (\%)

\begin{tabular}{|c|c|c|}
\hline Age (mean (SD)) & $54(16)$ & - \\
\hline Male $\mathrm{n}(\%)$ & $282(50.5)$ & - \\
\hline Hypertension n (\%) & $304(54.5)$ & - \\
\hline Diabetes n (\%) & $227(40.7)$ & - \\
\hline Cardiovascular disease n (\%) & $75(13.4)$ & - \\
\hline Chronic kidney disease n (\%) & $54(9.7)$ & - \\
\hline Cancer in past five years $\mathrm{n}(\%)$ & $13(2.3)$ & - \\
\hline Overweight n (\%) & $124(22.2)$ & - \\
\hline General ward n (\%) & $418(75.9)$ & $7(1.3)$ \\
\hline High Care n (\%) & $85(15.4)$ & $7(1.3)$ \\
\hline ICU n (\%) & $48(8.7)$ & $7(1.3)$ \\
\hline Died n (\%) & $121(21.9)$ & $6(1.1)$ \\
\hline Received steroids n (\%) & $432(78 \%)$ & $7(1.3)$ \\
\hline HIV positive n (\%) & $82(14.7)$ & - \\
\hline Past TB diagnosis n (\%) & $25(4.5)$ & - \\
\hline Active TB diagnosis $\mathrm{n}(\%)$ & $14(2.5)$ & - \\
\hline Days hospitalised (median [IQR]) & $6[3-10]$ & - \\
\hline Respiratory rate bpm (median [IQR]) & $22[20-25]$ & $21(3.8)$ \\
\hline Estimated $\mathrm{FiO}_{2} \%$ (median [IQR]) & $0.37[0.21-0.70]$ & $26(4.7)$ \\
\hline Peripheral $\mathrm{O}_{2}$ saturation $\%$ (median [IQR]) & $93[90-96]$ & $15(2.7)$ \\
\hline ROX score (median [IQR]) & $8.2[4.8-16.7]$ & $33(5.9)$ \\
\hline Systolic blood pressure mm/Hg (median [IQR]) & $123[111-136]$ & $18(3.2)$ \\
\hline ANC $\times 10^{-9} / \mathrm{L}$ (median [IQR]) & $6.7[4.8-10.0]$ & $159(28.5)$ \\
\hline ALC $\times 10^{-9} / L$ (median [IQR]) & $1.1[0.8-1.6]$ & $159(28.5)$ \\
\hline NLR (median [IQR]) & $6.2[3.4-9.8]$ & $159(28.5)$ \\
\hline CRP mg/L (median [IQR]) & $108[46-181]$ & $82(14.7)$ \\
\hline Ferritin $\mu \mathrm{g} / \mathrm{L}$ (median [IQR]) & $527[238-1171]$ & $150(26.9)$ \\
\hline PCT $\mu \mathrm{g} / \mathrm{L}$ (median [IQR]) & $0.13[0.05-0.58]$ & $244(43.7)$ \\
\hline
\end{tabular}




\begin{tabular}{lll}
\hline DDIM mg/L (median [IQR]) & $0.88[0.39-1.95]$ & $113(20.2)$ \\
\hline HbA1c \% (median [IQR]) & $7.2[6.3-10.3]$ & $292(52.3)$ \\
\hline Creatinine (median [IQR]) & $84[67-115]$ & $64(11.5)$ \\
\hline ALT (median [IQR]) & $32[20-54]$ & $130(23.3)$
\end{tabular}

Vital signs and laboratory biomarker levels measured at admission ( \pm 24 hours). SD = standard deviation. $\mathrm{n}$ = sample size. $\mathrm{IQR}=$ interquartile range. bpm = beats per minute. $\mathrm{FiO}_{2}=$ fraction of inspired oxygen. ROX = respiratory oxygenation score. $A N C=$ absolute neutrophil count. ALC = absolute lymphocyte count. $\mathrm{NLR}=$ neutrophil: lymphocyte ratio. $\mathrm{CRP}=\mathrm{C}$-reactive protein. $\mathrm{PCT}=$ procalcitonin. $\mathrm{DDIM}=\mathrm{D}$-dimer. $\mathrm{ALT}=$ alanine aminotransferase.

\section{ROX scores and laboratory biomarkers are associated with COVID-19 severity and mortality}

The median ROX score at admission was 8.2 (IQR: 4.8-16.7). Increasing age was associated with lower ROX scores at admission (correlation of age and ROX score: rho $=-0.2, P<0.001$ ), and higher DDIM and inflammatory biomarker levels (Figure 2A-B). ROX scores, inflammatory biomarkers and DDIM levels showed significant collinearity with each other (Figure 2B).

A total of 85 patients required High Care $(\mathrm{HC})$ as their highest level of supportive care and 48 patients needed Intensive Care Unit (ICU) admission. Length of hospitalisation was longer for those admitted to ICU than the general wards (median 16 days vs 5 days, $P<0.001$ ). In addition, patients admitted to ICU had more severe disease - ROX scores were lower, ANC, PCT, DDIM and ALT levels were significantly higher when compared to patients admitted to HC or general wards (Table S1). Consequently, higher ROX scores, ANC, NLR and PCT levels predicted admission to ICU with AUROC > 0.7. ROX scores below 4.5 had the highest specificity at $82 \%$ (sensitivity $62 \%$ ), and PCT above 0.13 the highest sensitivity at $94 \%$ (specificity $57 \%$ ). The mortality rate was $57 \%$ for patients admitted to ICU, $24 \%$ for High Care, and $17 \%$ for general ward admissions (FET $P<0.001$ for ICU vs HC or General wards).

A total of $121(21.7 \%)$ patients died during admission. Increasing age was associated with increased mortality during the admission (Figure 2C). A ROX score below six at admission was associated with a two-fold increase in mortality compared to higher ROX scores (aHR $=2.1,95 \% \mathrm{Cl}: 1.2-3.6, P=0.01)$. The tertiles grouping the highest levels of creatinine, CRP, ferritin, NLR and DDIM were also associated with increased mortality (Figure 3C). Sex and diabetes were not associated with increased mortality during hospitalisation, but chronic kidney disease was (aHR $=2.4,95 \% \mathrm{Cl}: 1.5-3.9, P<0.001)$. HIV was not associated with significantly increased mortality (aHR $=1.1,95 \% \mathrm{Cl}$ : 0.6-2.0, $P=0.14$ ) (Figure 2C).

\section{COVID-19 severity in people living with HIV}

Respiratory rates were equivalent between PWH and HIV-negative patients, however PWH needed less oxygen at admission, and had higher peripheral oxygen saturation readings. ROX scores were higher in PWH, but the difference was not significant (Table 2). There was also no significant difference in NLR, 
CRP, ferritin, PCT, DDIM, ALT, or HbA1C\% levels between PWH and HIV-negative patients. Creatinine levels were slightly lower in $\mathrm{PWH}$, when compared to HIV-negative patients. These associations were unchanged in linear regression models which adjusted for age differences in HIV-negative patients and PWH. PWH were as likely as HIV-negative patients to require admission to ICU - 3.7\% of PWH vs $9.5 \%$ HIV-negative patients admitted to ICU (FET $P=0.2$ ).

\section{Markers of disease severity in people living with HIV, stratified by CD4 count and HIV-1 viral load}

In PWH, a CD 4 count below 200 cells/ $\mu \mathrm{L}$ was associated with lower odds of having hypertension, diabetes or being on ART (Table S2). PWH with CD 4 counts below 200 cells/ $\mu \mathrm{L}$ had higher HIVVLs, NLRs and DDIM levels (Figure 3 A) and were more likely to have TB ( $25 \%$ vs $4 \%, P=0.06)$ when compared to PWH with higher CD4 counts. ROX scores were equivalent between those with higher and lower CD4 counts (Figure 4A). Admission rates to $\mathrm{HC}$ or ICU were equivalent between $\mathrm{PWH}$ with $\mathrm{CD} 4$ counts above or below 200 cells $/ \mu \mathrm{L}$.

PWH with detectable HIVVLs were less likely to be on ART (OR $=0.11,95 \% \mathrm{Cl}: 0.01-0.59, P=0.003)$, and had lower median CD4 counts when compared to those with suppressed HIVVLs (34 [IQR: 16-47] vs 256 [IQR: 134-429], MW P<0.0001) (Table S3). PWH with detectable HIVVLs were younger than those with suppressed HIVVLs (mean age 40 years vs 48 years, $T$ test $P=0.02$ ) and were less likely to have diabetes (OR $=0.12,95 \% \mathrm{Cl}: 0.003-0.97, P=0.04)$. PWH with detectable HIVVLs had significantly higher ROX scores that those with a suppressed HIVVLs (17.8 [IQR: 10.9-20.5] vs 6.7 [IQR: 4.3-10.1], MW P=0.005). Laboratory biomarker levels were not associated with HIVVL (Figure 3C). None of the PWH with detectable HIVVLs were admitted to HC or ICU.

Table 2: Summary and comparison of admission variables by HIV status 
Age years (mean (SD))

Male n (\%)

Hypertension $\mathrm{n}(\%)$

Diabetes n (\%)

Cardiovascular disease n (\%)

Chronic kidney disease $\mathrm{n}(\%)$

Cancer in past five years $\mathrm{n}(\%)$

Overweight n (\%)

General ward n (\%)

High Care n (\%)

ICU n (\%)

Died n (\%)

Received steroids n (\%)

Previous TB diagnosis n (\%)

Active TB diagnosis $\mathrm{n}(\%)$

Days hospitalised (median [IQR])

Respiratory rate bpm (median [IQR])

Estimated $\mathrm{FiO}_{2}$ (median [IQR])

Peripheral $\mathrm{O}_{2}$ saturation (median [IQR])

ROX score (median [IQR])

Systolic blood pressure $\mathrm{mm} / \mathrm{Hg}$ (median [IQR])

ANC $\times 10^{-9} / \mathrm{L}$ (median [IQR])

ALC $\times 10^{-9} / \mathrm{L}$ (median [IQR])

NLR (median [IQR])

CRP mg/L (median [IQR])

Ferritin $\mu \mathrm{g} / \mathrm{L}$ (median [IQR])

PCT $\mu \mathrm{g} / \mathrm{L}$ (median [IQR])
HIV-negative

$n=476$

PWH

$\mathrm{n}=\mathbf{8 2}$

$56( \pm 16)$

243 (51.1)

275 (57.8)

200 (42.0)

73 (15.3)

47 (9.9)

10 (2.1)

111 (23.3)

354 (75.3)

71 (15.1)

45 (9.5)

109 (23.1)

372 (78.2)

7 (1.5)

5 (1.1)

6 [3-10]

22 [20 - 25]

$0.40[0.21-0.70]$

93 [90 - 95]

$7.9[4.7-15.9]$

124 [112 - 137]

7.0 [4.4 - 10.5]

$1.2[0.8-1.6]$

6.2 [3.4 - 10.3]

108 [47 - 181]

520 [232 - 1232]

$0.14[0.05-0.59]$

Page $11 / 23$
$46( \pm 12)$

$<0.001$

39 (47.6)

0.63

29 (35.4)

$<0.001$

27 (32.9)

0.14

2 (2.4)

0.001

7 (8.5)

0.84

3 (3.7)

0.42

13 (15.9)

0.15

64 (79.0)

0.2

14 (17.3)

3 (3.7)

12 (14.8)

0.11

$60(74.0)$

0.31

18 (22.0)

$<0.001$

$9(11.0)$

$<0.001$

6 [3-10]

0.73

22 [18 - 25]

0.85

$0.22[0.21-0.58] \quad 0.07$

94 [91 - 96]

0.02

$9.8[5.5-19.5]$

0.21

118 [105 - 130]

0.001

$6.2[4.2-9.2]$

0.18

$1.1[0.7-1.6]$

0.54

$6.0[3.5-9.2]$

0.81

110 [42 - 183]

0.99

542 [295 - 1028] 0.87

$0.08[0.04-0.46] \quad 0.33$ 


\begin{tabular}{llll}
\hline DDIM mg/L (median [IQR]) & $0.88[0.39-1.95]$ & $1.03[0.36-2.08]$ & 0.86 \\
\hline HbA1c \% (median [IQR]) & $7.1[6.3-10.3]$ & $7.7[6.2-12.4]$ & 0.39 \\
\hline Creatinine $\mu$ mol/L (median [IQR]) & $85[68-115]$ & $78[61-103]$ & 0.03 \\
\hline ALT U/L (median [IQR]) & $33[20-54]$ & $31[19-47]$ & 0.40
\end{tabular}

Vital signs and biomarker levels measured at admission ( \pm 24 hours). $P$ values shown for FET for categorical variables, $\mathrm{T}$ test (age comparison) and $\mathrm{MW}$ test (all other continuous variables). $\mathrm{PWH}=$ People living with HIV. SD = standard deviation. $\mathrm{n}=$ sample size. IQR = interquartile range. $\mathrm{FiO}_{2}=$ fraction of inspired oxygen. $\mathrm{ROX}=$ respiratory oxygenation score. $\mathrm{ANC}=$ absolute neutrophil count. $\mathrm{ALC}=$ absolute lymphocyte count. NLR = neutrophil: lymphocyte ratio. $\mathrm{CRP}=\mathrm{C}$-reactive protein. $\mathrm{PCT}=$ procalcitonin. DDIM = D-dimer. $\mathrm{ALT}=$ alanine aminotransferase .

\section{Variables associated with mortality by HIV status}

In total, 109/476 (23.1\%) HIV-negative patients died during admission. Among HIV-negative patients, those who died were older (mean age 62 vs 52 years, $T$ test $P<0.001$ ), and more likely to have hypertension $(P<0.001)$, diabetes $(P=0.06), \mathrm{CVD}(P<0.001)$, or CKD $(P<0.001)$. Respiratory disease severity was significantly worse at admission in HIV-negative patients who died versus those who survived (ROX score: 4.8 vs 9.5 , MW $P<0.001)$. Mortality in the HIV-negative patients was also associated with higher levels of laboratory biomarkers, higher ANC, lower ALC and higher creatinine levels (MW $P<0.001$ ).

A total of 12/82 (14.8\%) PWH died during admission. PWH who died all had significant HIV-related comorbidities, or other risk factors for COVID-19-related mortality (Table 3). Their hospital stay was shorter than those who survived (median of 3 vs 7 days), and HIVVL and CD 4 counts were not associated with in-hospital mortality in univariate analyses (Log rank $P>0.05$ ) (Figure 3B \& D). Creatinine, CRP, PCT and DDIM levels were higher at admission in PWH who died compared to those who survived $(P<0.05$ for all comparisons). When compared to HIV-negative patients who died, $\mathrm{PWH}$ who died had higher DDIM levels at admission (2.3 [IQR: 1.6-5.9] vs 1.5 [IQR:0.9-3.4], MW $P=0.05$ ), were younger (mean age 49 vs 64, T test $P=0.001)$, less likely to be hypertensive (OR=0.23, $P=0.02)$ and more likely to have TB $(16.7 \%$ vs $0.9 \%, \mathrm{OR}=20.3, P=0.03$ ).

Table 3: Clinical description of PWH who died during admission 


\begin{tabular}{|c|c|c|c|c|c|c|c|}
\hline Patient & Age & Sex & Comorbidities & $\begin{array}{l}\text { CD4 count } \\
\text { (cells/ } / \mu \mathrm{L})\end{array}$ & $\begin{array}{l}\text { HIV-1 viral } \\
\text { load (IU/mL) }\end{array}$ & $\begin{array}{l}\text { ROX } \\
\text { score } \\
1\end{array}$ & $\begin{array}{l}\mathrm{HbA1c} \\
\%\end{array}$ \\
\hline 1 & 70 & Male & Hypertension & 375 & LDL & 1.9 & - \\
\hline 2 & 39 & Female & Overweight & 36 & LDL & 3.0 & 6.4 \\
\hline 3 & 35 & Female & Active TB ${ }^{2}$ & 106 & - & 15.6 & - \\
\hline \multirow[t]{2}{*}{4} & 47 & Male & Diabetes & 387 & LDL & 2.7 & 6 \\
\hline & & & $\begin{array}{l}\text { B-cell lymphoblastic } \\
\text { leukaemia }\end{array}$ & & & & \\
\hline 5 & 54 & Male & CKD stage 5 & - & - & - & 6.8 \\
\hline 6 & 48 & Female & Suspected SLE ${ }^{3}$ & 608 & LDL & 12.5 & - \\
\hline \multirow[t]{2}{*}{7} & 61 & Male & CKD stage 5 & 159 & - & 5.7 & - \\
\hline & & & Epilepsy & & & & \\
\hline \multirow[t]{5}{*}{8} & 28 & Male & Chronic HBV & 20 & 3310 & 10.2 & - \\
\hline & & & $\mathrm{HBV}$ viral load $=$ & & & & \\
\hline & & & $64113 \mathrm{IU} / \mathrm{mL}$ & & & & \\
\hline & & & B cell lymphoma & & & & \\
\hline & & & $\begin{array}{l}\text { Active TB on } \\
\text { treatment at } \\
\text { admission }\end{array}$ & & & & \\
\hline \multirow[t]{4}{*}{9} & 63 & Female & Hypertension & 612 & - & 3.2 & 15.7 \\
\hline & & & Diabetes & & & & \\
\hline & & & Obesity & & & & \\
\hline & & & CKD stage 3 & & & & \\
\hline \multirow[t]{4}{*}{10} & 40 & Female & Disseminated CMV & 44 & $1.36 \times 10^{6}$ & 20.6 & - \\
\hline & & & CMV viral load = & & & & \\
\hline & & & $27000 \mathrm{lU} / \mathrm{mL}$ & & & & \\
\hline & & & $\begin{array}{l}\text { Cavitating } \\
\text { pneumonia }\end{array}$ & & & & \\
\hline \multirow[t]{2}{*}{11} & 57 & Male & Hypertension & 577 & LDL & 8.3 & 13.3 \\
\hline & & & Diabetes & & & & \\
\hline 12 & 51 & Female & Hypertension & 429 & LDL & - & 6.1 \\
\hline
\end{tabular}


${ }^{1}$ ROX score at admission. ${ }^{2}$ TB treatment started on empirical grounds. ${ }^{3}$ Anti-nuclear antibody positive, with bicytopenia, rash and joint pain - admitted for haemoptysis and developed nosocomial SARS-CoV-2 infection. ROX = respiratory oxygenation score. $\mathrm{CKD}=$ chronic kidney disease. $\mathrm{HBV}=$ Hepatitis $\mathrm{B}$ virus. $\mathrm{CMV}=$ Cytomegalovirus. $\mathrm{LDL}=$ lower than detectable level. - indicates missing values

\section{Discussion}

We report a detailed analysis of clinical phenotypes of COVID-19 in hospitalised patients, with and without HIV, and their association with laboratory biomarkers.

PWH had similar levels of COVID-19 severity, whether estimated by levels of supportive care during hospitalisation, ROX scores or laboratory biomarkers when compared to HIV-negative patients. PWH were younger, less likely to be hypertensive, and had lower creatinine levels at admission. PWH with detectable HIVVLs had less severe respiratory disease, with equivalent levels of systemic inflammation as those with suppressed HIVVLs. Similar results have been reported in hospital cohorts from higher income settings $(7,30)$. PWH with suppressed HIVVLs were older, more likely to be on ART, and more likely to have other comorbidities when compared to those with detectable HIVVLs. The prevalence of comorbidities increased with age in this cohort, as has been reported in other studies (3). Therefore, it is possible that the association between respiratory disease severity and HIVVL was confounded by baseline differences in age and comorbidities in PWH, and not necessarily because of HIV viraemia. However, we should not dismiss the possibility that detectable HIV viral replication could alter immune phenotypes such that COVID-19 severity is affected.

Effective ART suppresses viral replication and reverses much of the immunopathology of HIV, but T cell and monocyte phenotypes, as well as levels of systemic immune activation, remain altered for years afterwards $(18,31)$. Monocyte, and cytokines involved in monocyte trafficking are central to the pathophysiology of COVID-19 (11). Monocytes are recruited to tissue by the interaction of their CCR2 receptor and its ligand CCL2, which is dysregulated in severe COVID-19 (9). HIV infection decreases CCR2 expression on monocytes, and this is reversed with suppressive ART (18). Reduced monocyte trafficking to lungs after SARS-CoV-2 infection may reduce later monocyte-derived inflammation in COVID-19, and this may be a mechanism to explain the less severe respiratory disease in PWH with detectable HIVVLs in this study. Currently reported COVID-19 studies have included few PWH with detectable HIVVLs, and more research is needed to determine the effect of HIV-infection on COVID-19 immune responses.

PWH who died often had significant coexisting comorbidities including active TB infection, lymphoma, disseminated cytomegalovirus, autoimmune disease, or other comorbidities, such as hypertension, diabetes, or CKD. PWH who died with suppressed viral loads were older, had higher CD 4 counts and had additional comorbidities like hypertension, obesity, and diabetes, while those with detectable HIVVLs had 
significant coinfections or HIV-associated malignancies. The sample size of PWH who died was small, and it is therefore difficult to draw statistically supported conclusions on these observations. Many of the larger studies which reported that HIV infection is associated with increased risk of severe COVID-19 and related mortality included a higher proportion of males in their analysis than our study $(3,32-34)$. It is possible that sex and HIV interact with COVID-19 to alter disease phenotypes. Neutrophils isolated from females have greater inflammatory responses to interferon, which may allow for better innate immune antiviral response (35). Males with severe COVID-19 have altered kynurenic acid metabolism which is associated weaker $\mathrm{T}$ cell responses (36). Further research should be undertaken to investigate the interaction of sex, age, and HIV infection on immune responses.

This analysis has several limitations: We analysed records for patients admitted to a single tertiary academic medical centre in an urban area, many patients are sent there by referral which may bias admission statistics towards those with more severe disease. Nevertheless, this cohort's prevalence of HIV-infection was within the range for other reported estimates and is likely to be broadly representative of similar hospital cohorts in South Africa.

Strengths of our study include prospective data collection, a validated method for quantifying respiratory disease severity on a continuum, many patients with laboratory biomarkers during admission, and $\mathrm{PWH}$ well characterised in terms of other comorbidities. We have generated several hypotheses related to HIV and COVID-19 for future exploration.

\section{Declarations}

\section{Data availability}

Complete individual patient data and the analysis code in $\mathrm{R}$ are available to researchers on reasonable request.

\section{We report no conflict of interests}

\section{This work was not supported by any specific research funding}

\section{Acknowledgements}

The authors would like to thank all the staff at the Steve Biko Academic Hospital and Tshwane District Hospitals who made it possible to collect these data.

\section{References}

1. Cohen C, Walaza S, Viboud C, Cohen AL, Madhi SA, Venter M, et al. Deaths Associated with Respiratory Syncytial and Influenza Viruses among Persons $\geq 5$ Years of Age in HIV-Prevalent Area, South Africa, 1998-2009 - Volume 21, Number 4-April 2015 - Emerging Infectious Diseases journal - CDC. [cited 2021 Aug 6]; Available from: https://wwwnc.cdc.gov/eid/article/21/4/14-1033_article 
2. Cohen C, Moyes J, Tempia S, Groome M, Walaza S, Pretorius M, et al. Mortality amongst Patients with Influenza-Associated Severe Acute Respiratory Illness, South Africa, 2009-2013. PLOS ONE. 2015 Mar 18;10(3):e0118884.

3. Jassat W, Cohen C, Tempia S, Masha M, Goldstein S, Kufa T, et al. Risk factors for COVID-19related in-hospital mortality in a high HIV and tuberculosis prevalence setting in South Africa: a cohort study. The Lancet HIV [Internet]. 2021 Aug 4 [cited 2021 Aug 6];0(0). Available from: https://www.thelancet.com/journals/lanhiv/article/PIIS2352-3018(21)00151-X/abstract

4. Boulle A, Davies M-A, Hussey H, Ismail M, Morden E, Vundle Z, et al. Risk factors for COVID-19 death in a population cohort study from the Western Cape Province, South Africa. Clin Infect Dis. 2020 Aug 29;

5. WHO Global Clinical Platform for COVID-19. Clinical features and prognostic factors of COVID-19 in people living with HIV hospitalized with suspected or confirmed SARS-CoV-2 infection [Internet]. World Health Organization; 2021. Available from: https://apps.who.int/iris/bitstream/handle/10665/342697/WHO-2019-nCoV-Clinical-HIV-2021.1-eng.pdf

6. Venturas J, Zamparini J, Shaddock E, Stacey S, Murray L, Richards GA, et al. Comparison of outcomes in HIV-positive and HIV-negative patients with COVID-19. Journal of Infection [Internet]. 2021 May 26 [cited 2021 Jun 6];0(0). Available from: https://www.journalofinfection.com/article/S01634453(21)00262-0/abstract

7. Patel VV, Felsen UR, Fisher M, Fazzari MJ, Ginsberg MS, Beil R, et al. Clinical Outcomes and Inflammatory Markers by HIV Serostatus and Viral Suppression in a Large Cohort of Patients Hospitalized With COVID-19. J Acquir Immune Defic Syndr. 2021 Feb 1;86(2):224-30.

8. Calligaro GL, Lalla U, Audley G, Gina P, Miller MG, Mendelson M, et al. The utility of high-flow nasal oxygen for severe COVID-19 pneumonia in a resource-constrained setting: A multi-centre prospective observational study. EClinicalMedicine. 2020 Nov;28:100570.

9. Consortium Co-19 MBAt (COMBAT), Ahern DJ, Ai Z, Ainsworth M, Allan C, Allcock A, et al. A blood atlas of COVID-19 defines hallmarks of disease severity and specificity. medRxiv. 2021 May $11 ; 2021.05 .11 .21256877$.

10. Thwaites RS, Sanchez Sevilla Uruchurtu A, Siggins MK, Liew F, Russell CD, Moore SC, et al. Inflammatory profiles across the spectrum of disease reveal a distinct role for GM-CSF in severe COVID19. Sci Immunol. 2021 Mar 10;6(57):eabg9873.

11. Vanderbeke L, Van Mol P, Van Herck Y, De Smet F, Humblet-Baron S, Martinod K, et al. Monocytedriven atypical cytokine storm and aberrant neutrophil activation as key mediators of COVID-19 disease severity. Nat Commun. 2021 Jul 5;12(1):4117. 
12. Hanna SJ, Codd AS, Gea-Mallorqui E, Scourfield DO, Richter FC, Ladell K, et al. T cell phenotypes in COVID-19 - a living review. Oxford Open Immunology [Internet]. 2021 Jan 1 [cited 2021 Feb 20];2(iqaa007). Available from: https://doi.org/10.1093/oxfımm/iqaa007

13. Mueller AA, Tamura T, Crowley CP, DeGrado JR, Haider H, Jezmir JL, et al. Inflammatory Biomarker Trends Predict Respiratory Decline in COVID-19 Patients. Cell Rep Med. 2020 Oct 29;1(8):100144.

14. Roca O, Messika J, Caralt B, García-de-Acilu M, Sztrymf B, Ricard J-D, et al. Predicting success of high-flow nasal cannula in pneumonia patients with hypoxemic respiratory failure: The utility of the ROX index. J Crit Care. 2016 Oct;35:200-5.

15. Results - RECOVERY Trial [Internet]. [cited 2021 Sep 25]. Available from: https://www.recoverytrial.net/results

16. Moir S, Fauci AS. B cells in HIV infection and disease. Nat Rev Immunol. 2009 Apr;9(4):235-45.

17. Walker B, McMichael A. The T-Cell Response to HIV. Cold Spring Harb Perspect Med. 2012 Nov;2(11):a007054.

18. McCausland MR, Juchnowski SM, Zidar DA, Kuritzkes DR, Andrade A, Sieg SF, et al. Altered Monocyte Phenotype in HIV-1 Infection Tends to Normalize with Integrase-Inhibitor-Based Antiretroviral Therapy. PLOS ONE. 2015 Oct 2;10(10):e0139474.

19. Riou C, Bruyn E du, Stek C, Daroowala R, Goliath RT, Abrahams F, et al. Profile of SARS-CoV-2specific CD4 T cell response: Relationship with disease severity and impact of HIV-1 and active Mycobacterium tuberculosis co-infection [Internet]. Infectious Diseases (except HIV/AIDS); 2021 Feb [cited 2021 Mar 2]. Available from: http://medrxiv.org/lookup/doi/10.1101/2021.02.16.21251838

20. Karim F, Gazy I, Cele S, Zungu Y, Krause R, Bernstein M, et al. HIV status alters disease severity and immune cell responses in $\beta$ variant SARS-CoV-2 infection wave [Internet]. 2021 Sep [cited 2021 Sep 27] p. 2020.11.23.20236828. Available from:

https://www.medrxiv.org/content/10.1101/2020.11.23.20236828v2

21. Snyman J, Hwa S-H, Krause R, Muema D, Reddy T, Ganga Y, et al. Similar antibody responses against SARS-CoV-2 in HIV uninfected and infected individuals on antiretroviral therapy during the first South African infection wave. Clinical Infectious Diseases [Internet]. 2021 Sep 2 [cited 2021 Sep 28]; (ciab758). Available from: https://doi.org/10.1093/cid/ciab758

22. Barbera LK, Kamis KF, Rowan SE, Davis AJ, Shehata S, Carlson JJ, et al. HIV and COVID-19: review of clinical course and outcomes. HIV Res Clin Pract. :1-17.

23. Kim H, Tanser F, Tomita A, Vandormael A, Cuadros DF. Beyond HIV prevalence: identifying people living with HIV within underserved areas in South Africa. BMJ Global Health. 2021 Apr 1;6(4):e004089. 
24. Simbayi L, Zuma K, Zungu N, Moyo S, Marinda E, Jooste S, et al. South African National HIV Prevalence, Incidence, Behaviour and Communication Survey, 2017: towards achieving the UNAIDS 90-9090 targets. 2019 [cited 2021 Oct 17]; Available from:

https://repository.hsrc.ac.za/handle/20.500.11910/15052

25. RStudio Team. RStudio: Integrated Development for R [Internet]. RStudio, PBC, Boston, MA; 2020. Available from: http://www.rstudio.com

26. Alboukadel Kassambara, Marcin Kosinski, Przemyslaw Biecek. survminer: Drawing Survival Curves using 'ggplot2'. R package version 048 [Internet]. Available from: https://CRAN.Rproject.org/package=survminer

27. Dardis C. survMisc: Miscellaneous Functions for Survival Data [Internet]. 2018. Available from: https://CRAN.R-project.org/package=survMisc

28. Alboukadel Kassambara. ggpubr: 'ggplot2' Based Publication Ready Plots. R package version 040 [Internet]. 2020; Available from: https://CRAN.R-project.org/package=ggpubr

29. Yoshida K, Bartel A, Chipman JJ, Bohn J, McGowan LDa, Barrett M, et al. tableone: Create 'Table 1' to Describe Baseline Characteristics with or without Propensity Score Weights [Internet]. 2021 [cited 2021 Dec 9]. Available from: https://CRAN.R-project.org/package=tableone

30. Durstenfeld MS, Sun K, Ma Y, Rodriguez F, Secemsky EA, Parikh RV, et al. Impact of HIV Infection on COVID-19 Outcomes Among Hospitalized Adults in the U.S. medRxiv. 2021 Apr 7;2021.04.05.21254938.

31. Cao W, Mehraj V, Kaufmann DE, Li T, Routy J-P. Elevation and persistence of CD8 T-cells in HIV infection: the Achilles heel in the ART era. Journal of the International AIDS Society. 2016 Jan;19(1):20697.

32. Sun J, Patel RC, Zheng Q, Madhira V, Olex AL, Islam JY, et al. COVID-19 Disease Severity among People with HIV Infection or Solid Organ Transplant in the United States: A Nationally-representative, Multicenter, Observational Cohort Study [Internet]. 2021 Jul [cited 2021 Oct 20] p. 2021.07.26.21261028. Available from: https://www.medrxiv.org/content/10.1101/2021.07.26.21261028v1

33. Tesoriero JM, Swain C-AE, Pierce JL, Zamboni L, Wu M, Holtgrave DR, et al. COVID-19 Outcomes Among Persons Living With or Without Diagnosed HIV Infection in New York State. JAMA Netw Open. 2021 Feb 1;4(2):e2037069.

34. Nomah DK, Reyes-Urueña J, Díaz Y, Moreno S, Aceiton J, Bruguera A, et al. Sociodemographic, clinical, and immunological factors associated with SARS-CoV-2 diagnosis and severe COVID-19 outcomes in people living with HIV: a retrospective cohort study. The Lancet HIV [Internet]. 2021 Oct 13 
[cited 2021 Oct 17];0(0). Available from: https://www.thelancet.com/journals/lanhiv/article/PIIS23523018(21)00240-X/fulltext

35. Gupta S, Nakabo S, Blanco LP, O'Neil LJ, Wigerblad G, Goel RR, et al. Sex differences in neutrophil biology modulate response to type I interferons and immunometabolism. PNAS. $2020 \mathrm{Jul}$ 14;117(28):16481-91.

36. Cai Y, Kim DJ, Takahashi T, Broadhurst DI, Yan H, Ma S, et al. Kynurenic acid may underlie sexspecific immune responses to COVID-19. Science Signaling. 2021 Jul 6;14(690):eabf8483.

\section{Figures}


A

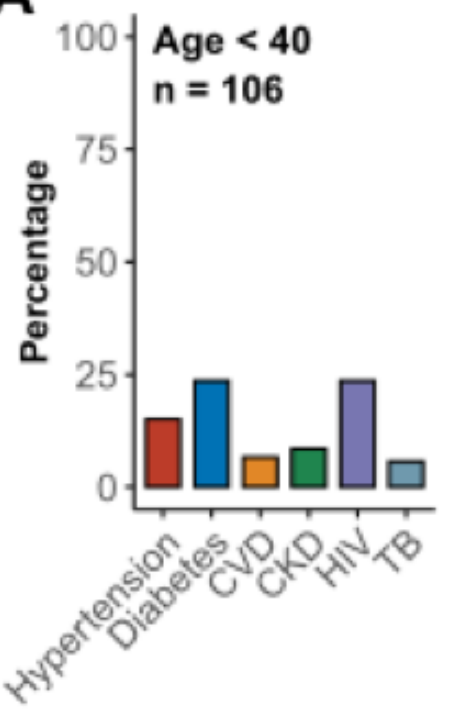

B
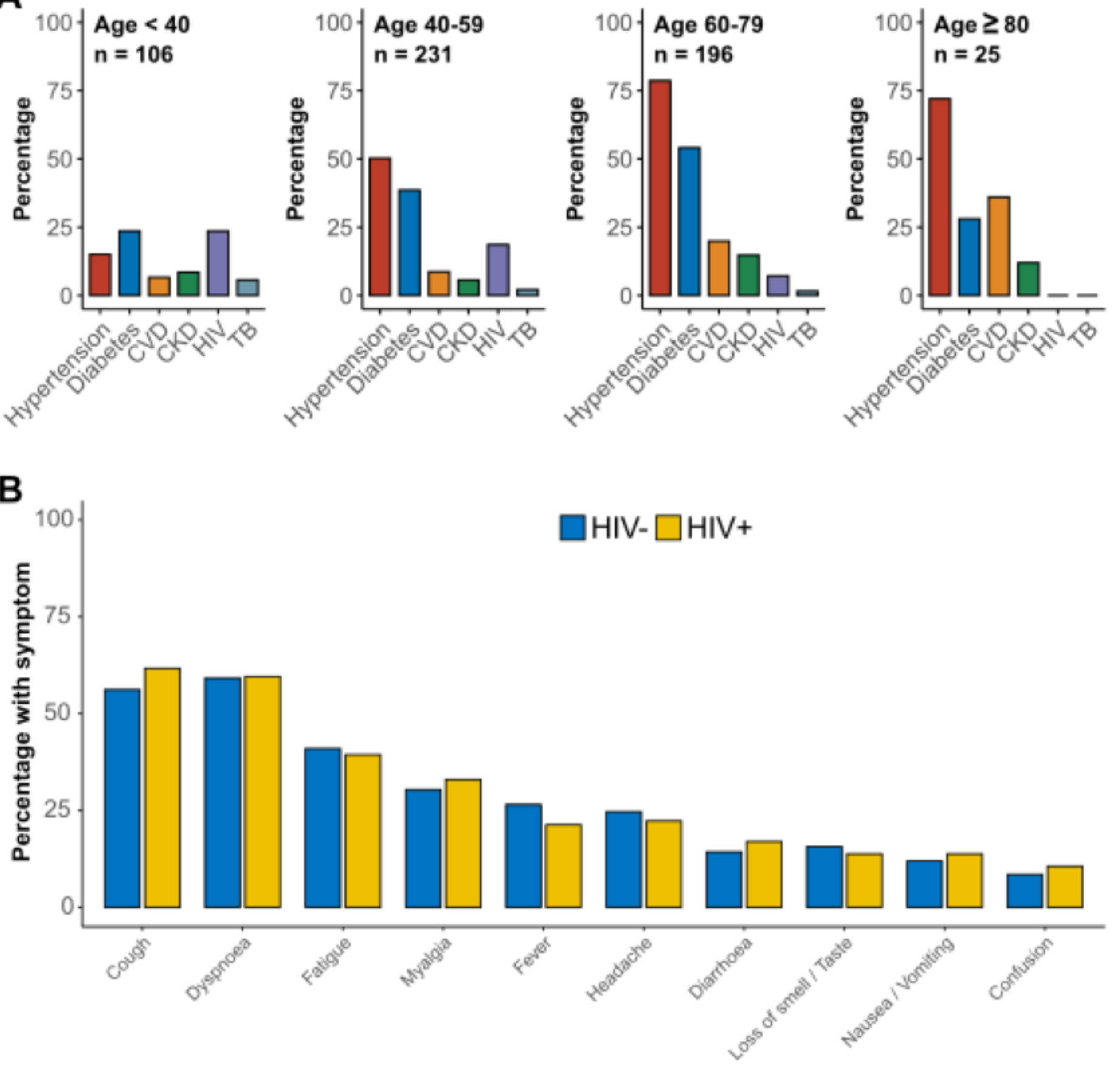

Figure 1

A) The prevalence of comorbidities by age categories is shown. HIV and diabetes were the most common comorbidities in patients younger than 40 years. Prevalence of non-communicable comorbidities increased with age, and HIV prevalence decreased. CVD = cardiovascular disease, CKD = chronic kidney disease. B) No significant differences in symptoms at admission by HIV status (Chi-squared $\mathrm{P}>0.05$ for all pairwise comparisons). Cough and dyspnoea were the most common symptoms at admission. 
A
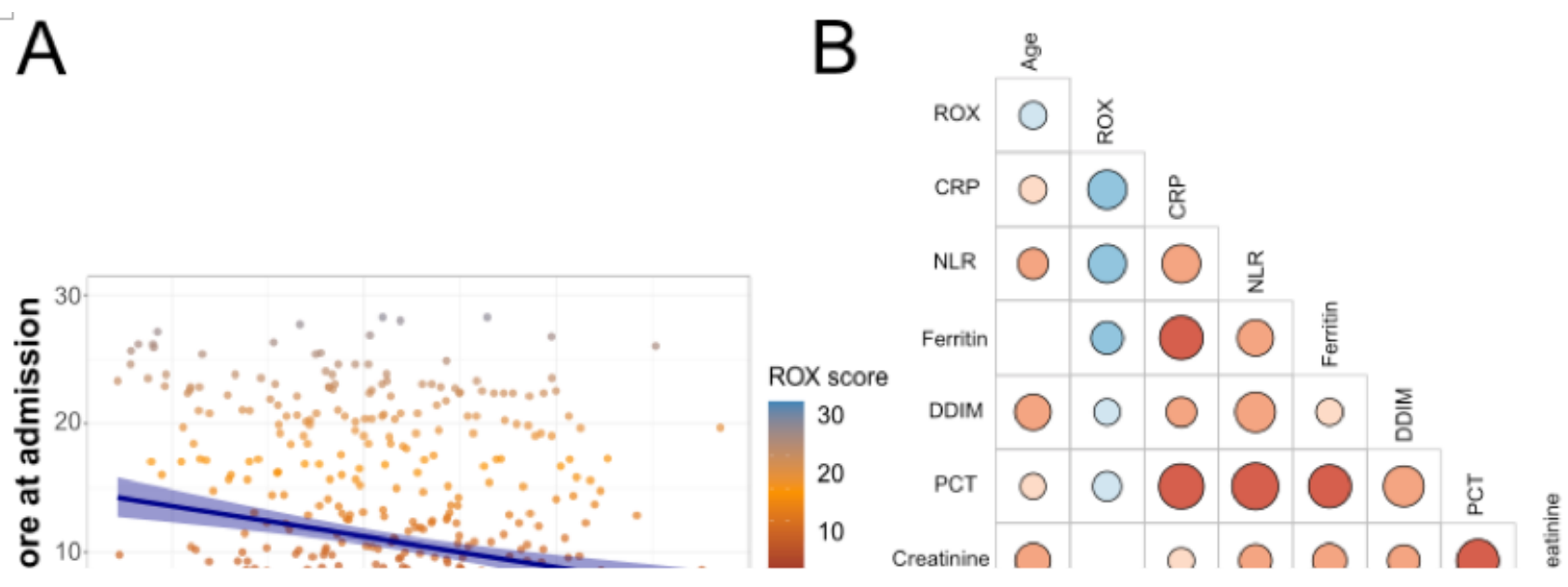

Figure 2

A) Age and ROX scores at admission correlated negatively (rho $=-0.2, P<0.001$ ). B) Correlation matrix of biomarkers with age and ROX score, non-significant correlations are shown as blank cells. The size and colour of the circles show the strength and direction of the Spearman correlation coefficients. C) Forest plot of the hazard ratio (HR) and their confidence intervals for variables association with in-hospital 
mortality. Age was associated with a strong effect on mortality; therefore, all other HRs are age-adjusted.

A

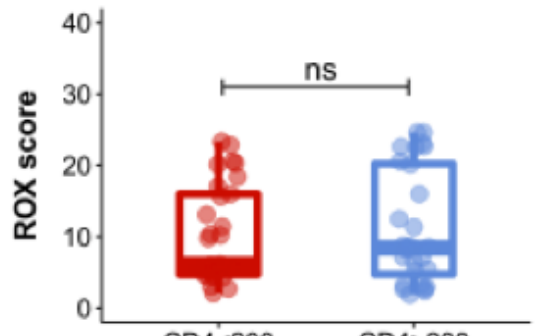

$\mathrm{CD} 4<200$

$\mathrm{CD} 4>200$

40
$-\quad 30$

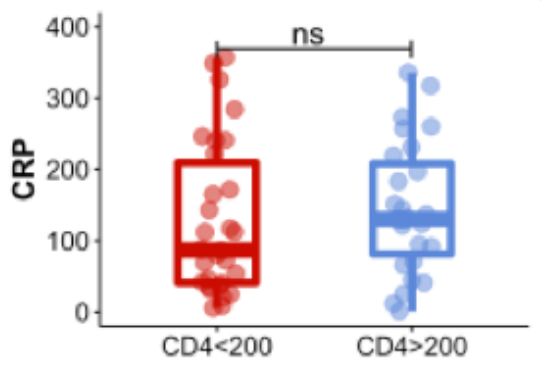

$40-1$
$-\quad 30-1$
B

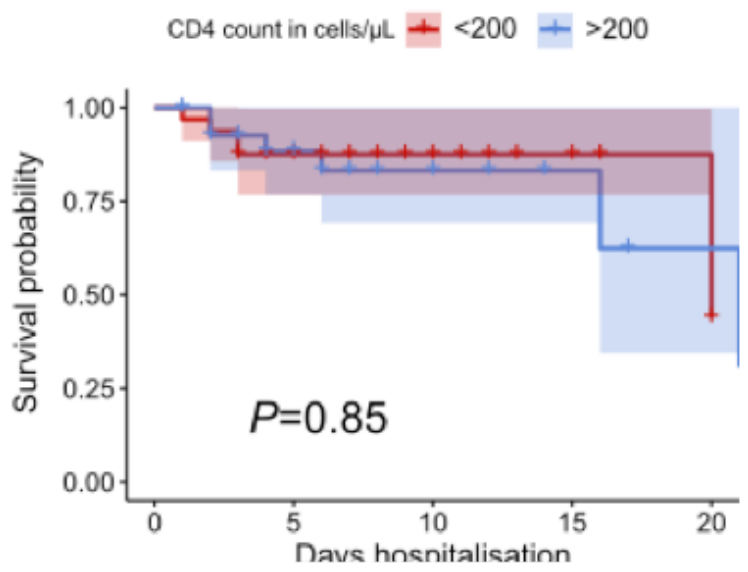

\section{Figure 3}

A) Pairwise comparisons for ROX scores, CRP, NLR and DDIM levels are shown for higher and lower CD4 counts. Lower CD4 counts were associated with higher NLR and DDIM levels at admission. B) KaplanMeier survival curves are shown for PWH stratified by CD4 count. There was no significant difference in time to death in hospital, logrank P value shown. C) Pairwise comparisons for ROX scores, CRP, NLR and D-dimer levels are shown for PWH by HIVVL. An HIVVL above 1000 copies/mL was associated with significantly higher ROX scores at admission. D) Kaplan-Meier survival curves overlapped for these patients indicating no significant difference in survival, logrank $P$ value shown. ${ }^{*}=P<0.05$; $* *=P<0.01$; ns $=$ not significant. 


\section{Supplementary Files}

This is a list of supplementary files associated with this preprint. Click to download.

- COVID19severityandinhospitalmortalityinanareawithhighHIVprevalencesupplementarymaterials.docx 